\title{
HANNIBAL LECTER COMO EJEMPLO DE PERSONAJE TRANSMEDIA: UN ESTUDIO DE CASO
}

\author{
José Manuel RUIZ MARTÍNEZ \\ Universidad de Granada \\ jmanuelruiz@ugr.es
}

\section{1}

\section{Introducción ${ }^{1}$}

Hannibal Lecter, «Hannibal el caníbal», es uno de los personajes más célebres del imaginario de ficción contemporáneo. Tanto su imagen, caracterizada ya de forma icónica por la máscara de hockey para prevenir sus mordeduras, como su personalidad, que mezcla un sadismo sin escrúpulos con un refinamiento cultural y gastronómico no exento de sorna, son algunas de las claves de la fascinación que genera entre el público. Como ha señalado el crítico Charles Gamlich,

[...] Thomas Harris [el creador del personaje] is not the only writer who helped create the myth of the modern serial killer... But no one has done it as well as Harris, and no serial killer has been so thoroughly trasformed into a cultural icon as has Hannibal Lecter. Particurlarly, no one but Harris has yet dared turn a serial killer into a hero (Simpson, 2010: 37).

Y, no obstante, la primera aparición de Hannibal Lecter es como un personaje secundario en la segunda novela de Harris. Después, paulatinamente, la figura va creciendo, cobrando protagonismo en las novelas sucesivas del autor y en las versiones cinematográficas de éstas — que se retroalimentan en la medida en que el éxito de las películas acaba condicionando la propia producción de las novelashasta dar el salto final la televisión. En el siguiente trabajo queremos plantear la naturaleza transmedial del personaje de Hannibal Lecter. Para ello, primero señalaremos el proceso progresivo de construcción de Lecter como creacción ficcional que atraviesa distintos medios. A continuación, discutiremos si se trata en efecto o no de un producto genuinamente transmedia, lo que nos parece que puede arrojar alguna luz, en cuanto estudio de un caso, a los límites y las taxonomías de la práctica transmedial (cfr. Scolari, 2012: 58), así como aportar una reflexión ulterior sobre alguna de sus características y singularidades más significativas.

\footnotetext{
${ }^{1}$ Este artículo se enmarca dentro del proyecto Narrativas transmediales: nuevos modos de ficción audiovisual, comunicación periodística y performance en la era digital (Referencia CSO2013-47288-P), dirigido por el profesor Domingo Sánchez-Mesa Martínez. Ministerio de Economía y Competitividad.
} 


\section{Hannibal: de personaje secundario en una novela a narrativa transmedia}

El personaje de Hannibal Lecter aparece por primera vez, como hemos dicho, en la segunda novela de Thomas Harris El dragón rojo (Red Dragon, 1981) ${ }^{2}$. Se trata de un personaje secundario - si bien necesario en el desarrollo de la trama-: un asesino en serie al que el protagonista de la novela, el detective Will Graham, ya detuvo en el pasado. De resultas de esta detención, Graham fue gravemente herido por Lecter y acabó retirado debido al trauma, no sólo físico. La acción de la novela comienza con la aparición de un nuevo psicópata que asesina familias enteras y practica con ellas extraños rituales. Ante la gravedad de los hechos, Jack Crawford visita a Graham en su retiro para pedirle que los ayude con el caso. En un momento dado, y ante la dificultad para comprender el perfil psicológico del asesino, a pesar del evidente trauma que le supone, Will Graham va a ver al Lecter al psiquiátrico donde se halla encerrado para inspirarse y que le ayude con el caso: «Quería tener una opinión. Un punto de vista muy extraño que necesitaba compartir; un enfoque que debía recobrar al cabo de esos apacibles años en los cabos» (Harris, 1992: 79). En la entrevista, Lecter presenta las características que más tarde lo harán famoso: encerrado en un entorno de máxima seguridad y después de haber sido advertidos de lo peligroso y cruel que es a través de una conversación entre Graham y el director del centro, se nos presenta leyendo Le Grand Dictionaire de Cuisine de Alexandre Dumas (Harris, 1992: 84). Lecter accede a examinar el expediente del caso y da algunas sugerencias esclarecedoras a Graham, pero no sin cobrarse su precio haciéndole preguntas insidiosas y enfrentando a éste con sus temores ocultos, que Hannibal sabe - haciendo honor a su nombre- leer o intuir. Tras la entrevista, Lecter aprovecha su derecho para usar el teléfono y hablar con su abogado y, a través de un ardid, consigue la dirección actual de Will y acaba por instar al psicópata a asesinar a Will junto con su mujer y su hijo. La novela está contada por un narrador omnisciente, objetivo, no focalizado en ningún personaje concreto: está en todas partes donde conviene - incluso es capaz de dar saltos muy atrás en el pasado - y nos lo cuenta todo de todos. Hannibal Lecter aparece solamente en los capítulos 7 - la entrevista - 8 - el ardid telefónico - y el 24, donde asistimos a una breve escena donde se le retiran todos los libros y otros materiales de entretenimiento de su celda como castigo por haber enviado inducido al psicópata a asesinar. En el capítulo 36 podemos leer una carta que le envía a Will acusándolo de propiciar y disfrutar de la muerte; de ser un psicópata como él: de ahí su habilidad para encontrarlos. Por último, en el capitulo 54, el final, Graham, en la unidad de cuidados intensivos, de nuevo muy gravemente herido, esta vez por el psicópata al que estaban investigando y al que también ha conseguido encontrar, Graham recibe una segunda carta de Lecter donde hurga sin piedad y con ironía en las heridas del detective. Quizá esta presencia de Lecter en el último capítulo sea el único indicio del interés del autor por el personaje.

La novela recibió buenas críticas dentro de su género - la novela negra-, entre otras la entusiasta de Stephen King (Simpson, 2010: 87) y se convirtió en un best seller que le reportó al autor

\footnotetext{
${ }^{2}$ Thomas Harris es un novelista estadounidense nacido en Jackson, Tennessee, en 1940. Antes de dedicarse exclusivamente a escribir ficciones fue reportero de casos criminales para distintos medios. Su primera novela, Domingo negro (Black Sunday, 1975) trata sobre los atentados terroristas de las olimpiadas de Munich de 1972, y fue llevada al cine por John Frankenheimer en 1975 (vid. Simpson, 2010: 1-45).
} 
más de un millón de dólares de beneficios (Simpson, 2010: 13-14). En 1983 adquirió los derechos para el cine el productor Dino de Laurentiis. La versión cinematográfica se tituló Manhunter (1986) y fue dirigida por Michael Mann, con Brian Cox en el papel de Hannibal Lecter — que en la película pasó a llamarse, un tanto absurdamente «Lektor»-. La película resultó un fracaso comercial, y Harris manifestó su descontento con el resultado - aunque la interpretación de Lecter de Brian Cox le gustó- (Simpson, 2010: 15). Por tanto, en su debut, el personaje de Lecter no pareció suscitar una atención especial y pasó desapercibido.

La tercera novela de Harris apareció en 1988: El silencio de los corderos (The Silence of the Lambs). En ella, el personaje de Hannibal Lecter comienza como secundario de una forma idéntica a El dragón rojo ${ }^{3}$ : ayuda a la protagonista, la joven agente Clarice Starling a atrapar a un nuevo psicópata, supuestamente a cambio de mejoras en su reclusión pero en realidad siguiendo sus propios intereses y su propia diversión, intentando enfrentar a Starling con sus peores sospechas y miedos al obligarla a revelar datos vitales íntimos a cambio de la ayuda con el perfil del asesino. No obstante, aquí la presencia de Lecter se intensifica. De nuevo con el mismo tipo de narrador omnisciente, la primera entrevista - y por ende la presencia de Lecter en la narración - ya se produce en el capítulo 3; hay una segunda en el capítulo 9. A partir de que, en este caso, se descubre que el doctor Lecter puede tener información empírica relevante del caso por las cosas que dice, la figura de Lecter cobra protagonismo: la investigación gira en torno a él y a cómo conseguir que hable. Tercera aparición y entrevista con Clarice Starling en el capítulo 22; cuarta en el 25. En el capítulo 26, la focalización del narrador omnisciente ya está centrada en Hannibal y, a partir de ahí, de sujeto pasivo, se torna activo: aprovecha la torpeza del Dr. Chilton, el director del hospital donde está recluido, y, en un traslado, consigue escapar; pero antes tiene una última entrevista con Starling - capítulo 35-. El resto de la novela es una carrera contra reloj para atrapar al asesino. Pero el capítulo 61, el último, nos presenta a Lecter disfrutando de una buena cena en una habitación de hotel. Por su forma de vida, había previsto las necesidades de una fuga con antelación (pasaporte falso, dinero); va a huir a Río de Janeiro para hacerse la cirugía estética pero, provisionalmente, se ha inyectado silicona en la nariz para modificar su aspecto. Ahí escribe una última carta a Starling para despedirse de ella. Llegados a este punto, Lecter se ha apropiado del protagonismo en la novela, que se convierte en un best seller instantáneo y cuya recepción crítica es incluso mejor que la de El dragón rojo; El silencio de los corderos ganará el premio Bram Stoker de 1989 otorgado por la Asociación Norteamericana de Escritores de Terror (Simpson, 2014: 15). Esta película recoge, además, un detalle presente en la novela que será una marca icónica de reconocimiento del personaje: la máscara de hockey que se le pone a Lecter en sus traslados para prevenir sus mordeduras.

Después del fracaso de Manhunter, el productor Dino de Laurentiis, que tenía la prelación de los derechos de las novelas de Harris, los cede — de lo que luego se arrepentiría- y son adquiridos por

\footnotetext{
${ }^{3}$ En la reedición de Red Dragon de 2000, Harris añade un prólogo donde dice: «Years later, when I started The Silence of the Lambs, I did not know that Dr. Lecter would return [...]. I began with Clarice Starling and, not two pages into the new novel, I found she had to go visit the doctor» (Harris, 2000: pos. 156).
} 
Orion (Simpson, 2010: 15). En 1991 aparece la versión cinematográfica de El silencio de los corderos, dirigida por Jonathan Demme, ahora con Anthony Hopkins en el papel de Hannibal Lecter. La película es un gran éxito de público: fue el tercer mejor estreno de 1991, con una recaudación de ciento treinta millones de dólares, y doscientos cincuenta millones más en el resto del mundo, y salvó a Orión de una ruina inminente (Simpson, 2010: 16). Las recensiones críticas también fueron muy favorables en todo el mundo. Además obtuvo cinco Oscars de Hollywod, los principales: mejor película, mejor director, mejor guión adaptado, mejor actriz principal — para Jodie Forster por el papel de Claricey mejor actor principal —no secundario— para Anthony Hopkins por Hannibal Lecter ${ }^{4}$.

No cabe duda de que El silencio de los corderos marca un punto de inflexión en el reconocimiento de Hannibal Lecter. El prestigioso novelista y ensayista británico John Lanchester resume fascinación que adquiere desde entonces el personaje:

Lecter is straightfowardly a monster, bearing no relation to any muderer who ever lived, but achieving an arechetypal quality through his brilliance and inexplicability, his mythic quality as instatly apparent as, say, Sherlock Holmes' (Simpson, 2010: 144).

Esta fascinación puede apreciarse en el hecho de que el propio Demme dijo en una entrevista en la propia ceremonia donde recogió el Oscar que estaba impaciente por rodar una secuela de la película (Simpson, 2010: 16).

La tercera novela de lo que ya era una saga apareció nada menos que once años después, en 1999, cuando ya se pensaba que Harris no escribiría ninguna secuela. El título es muy significativo: Hannibal. La novela es una continuación de lo sucedido en El silencio de los corderos y, aunque de nuevo Clarice Starling tiene un papel destacado como investigadora, el verdadero protagonista de la obra es Hannibal Lecter. Aquí ya no estamos ante el planteamiento de las novelas precedentes — la caza de un psicópata para impedir que siga asesinando_-, sino que nos encontramos con una suerte de thriller de aventuras donde se entremezcla el intento de varios actores —incluida Starling, pero no solo ella- de atrapar a Lecter, del que han aparecido indicios tras un prolongado silencio, con la venganza urdida contra él por una de sus víctimas supervivientes — pero horriblemente desfigurada-. Por lo demás, la novela abunda en la descripción del personaje y despliega todos los clichés por los que se reconoce: su cultura, su gusto por el $\operatorname{arte}^{5}$ y la gastronomía, su cualidad de bon vivant sumadas a su proverbial inteligencia, penetración y refinamiento en su crueldad sin escrúpulos. No obstante, para algunos críticos como Robert Winder, en esta novela se muestra en exceso al personaje, con lo que se le resta fascinación mítica, el carácter elusivo, vampírico, casi sobrenatural, de las representaciones

\footnotetext{
${ }^{4}$ Hopkins se convertiría en el primer actor en ganar un Oscar por interpretar a un villano desde Marlon Brando por El Padrino (The Godfather, 1992); y el primero en hacerlo por un papel en una película de terror desde Fredric March por Dr. Jekyll y Mr. Hyde (1932); además, solo dos películas habían ganado con anterioridad los cinco premios principales: Sucedió una noche (It Happened One Night, 1934), y Alguien voló sobre el nido del cuco (One Flew Over the Cuckoo's Nest (1975) (Simpson, 2010: 16).

${ }^{5}$ En la segunda parte de la novela, nos encontramos con Lecter viviendo en Florencia de incógnito con el nombre de Dr. Fell (caído en inglés, en una posible referencia - del propio Lecter, que ha escogido el nombre- al Diablo). Habla un perfecto italiano y consigue el puesto de conservador del Palazzo Caponi (tras la misteriosa desaparición de quien ocupaba antes el cargo) tras demostrar su asombrosa erudición sobre la historia y el arte de Florencia ante un comité de especialistas locales.
} 
precedentes: «The more we learn about Lecter, the less resonant he becomes. In gaining his freedom, in shopping for expensive soap and driving a vintage Jaguar, he loses the magical potency with which he outwitted the world's best efforts to restrain him» (Simpson, 2010: 205).

La novela también fue un best seller, con más de cuatro millones de ejemplares vendidos en la edición de bolsillo, aunque la crítica esta vez estuvo dividida acerca de la calidad de la obra (Simpson, 2010: 22, 204-208). La adaptación cinematográfica (Hannibal, 2001) comenzó a gestarse cuando aún no se había publicado ésta, a partir del manuscrito. Fue producida por Dino de Laurentiis - que seguía teniendo la prelación de los derechos - más MGM — que poseía ahora a Orion, que finalmente quebró- y Universal. Estaba previsto que fuera dirigida por Demme y protagonizada de nuevo por Jodie Foster y Anthony Hopkins; pero Demme no quiso participar en el proyecto porque no le gustó la historia, que consideró demasiado violenta: finalmente, el director fue Ridley Scott. Jodie Foster, aunque había manifestado su deseo de seguir interpretando a Starling, también acabó declinando la oferta; fue sustituida por Julianne Moore. Hopkins, tras diversas dudas, finalmente, encarnó de nuevo al doctor Lecter —de no haberlo hecho, el proyecto no habría seguido adelante (Simpson, 2010: 22)—. El aspecto más destacado de la adaptación es el cambio en la película del atrevido final de la novela por otro más convencional y comercial ${ }^{6}$ : La película fue un éxito de taquilla: ha tenido la mayor recaudación para el estreno de una película calificada en EE. UU. como «R» — para mayores de dieciocho años ${ }^{7}$ - y obtuvo ciento cuarenta y cuatro millones de dólares de recaudación en todo el mundo; no obstante, como sucedió con la novela, las críticas fueron encontradas (Simpson, 2010: 24).

Tras este éxito, Dino de Laurentiis decide en 2002 hacer un remake de El dragón rojo, de la que ya tenía los derechos, con objeto de explotar el éxito del personaje. Esta película, a diferencia de Manhunter, se realiza de forma deliberada debido al éxito del personaje, que pasa a ser central en el guion - aunque, como hemos visto, no lo sea en la novela original—, y además establece nexos con las otras películas a partir de El silencio de los corderos, comenzando por el fundamental: la presencia de Anthony Hopkins como Hannibal, quien perdió peso y se sometió a un régimen de ejercicio (Simpson, 2010: 27) — aparte de dejarse una desconcertante coleta — para interpretar un personaje que era años más joven que en sus dos anteriores encarnaciones.

En 2004 surgen los primeros rumores de una nueva novela de Harris sobre Hannibal Lecter, que, en efecto, va a ser una prolepsis de la historia — vulgo precuela-. Se filtran varios nombres, algunos de ellos delirantes ${ }^{8}$. En 2005, se anuncia el supuestamente definitivo: Behind the Mask. No obstante,

\footnotetext{
${ }^{6}$ En la novela, Clarice acaba por sucumbir a la fascinación de Lecter y asumir su propia naturaleza poco convencional, de la que Lecter la ha ido convenciendo en sus encuentros, aquélla que le impedirá triunfar en el mundo mediocre y competitivo del FBI, y se fuga con él y se convierte en su amante, con lo que de algún modo se acaba cumpliendo lo que los periódicos sensacionalistas decían insidiosamente de ella: que era la novia del monstruo o la novia de Frankenstein. Sin embargo, como decimos, parecía un final excesivamente anticonvencional para la película, por lo que se cambió por el de Lecter esposado a Starling y teniendo que elegir, para poder escapar antes de que llegue la policía, entre cortarle la mano a ella o cortarse la suya propia. La escena, que culmina sin que sepamos qué mano ha cortado Lecter —lo sabremos después-, tiene un tono íntimo y erótico — Lecter besa a Clarice- donde comprendemos su atracción por ella, sumado al hecho de que finalmente sacrifica su propia mano a la de ella: eso es lo único que queda del romance original.

${ }^{7} \mathrm{El}$ dato es de 2010.

${ }^{8}$ The Lecter Variations; The Blooding of Hannibal Lecter; Hannibal IV; y, atención, The Adventures of Young Hannibal (sic) (Simpson, 2010: 28).
} 
la novela no aparecerá hasta 2006 con el título de Hannibal Rising. Un dato importante del cambio de estrategia con respecto a las novelas precedentes es que la película sale simultáneamente a la novela, — solo con unos pocos meses de demora, en febrero de 2007, dirigida por Peter Webb y con Gaspard Ulliel como el joven Lecter - y, sobre todo, que por primera vez Thomas Harris firma el guión de la adaptación de una de sus novelas, por lo que participa activamente en el proceso de traducción intermedial. Otros signos de este cambio hacia un modelo multimedia más dinámico fueron que el capítulo 6 de la novela estuvo disponible online meses antes del lanzamiento de la novela y que el propio Harris grabó una versión completa del libro en audio que comercializó Random House Audio (Simpson, 2010: 29) .

Con todo, Hannibal Lecter da el salto definitivo a un tercer medio cuando, sin que haya una nueva novela del personaje en perspectiva, en 2013 Dino de Laurentiis decide producir, junto con AXN, una serie sobre éste con el título sin equívocos de Hannibal, donde se abunda en los rasgos refinados y culturalistas de Lecter y resalta sus cualidades de dandi y bon vivant. Está desarrollada por Bryan Fuller ${ }^{10}$ y basada, según figura en los títulos de crédito, en los personajes de The Red Dragon — con el actor Mads Mikkelsen en el papel de Lecter-: por tanto, se sitúa cronológicamente entre lo que se cuenta en Hannibal Rising y Red Dragon; en realidad, tan solo poco tiempo antes de ésta. Fuller tenía previstas siete temporadas: las tres primeras contendrían trama original —y por ende añadida-; en la cuarta temporada se recontaría El dragón rojo; en la quinta, El silencio de los corderos; en la sexta, Hannibal, y la séptima incluiría un desenlace de nuevo inédito y original para el personaje más allá de la película al recuperar el final original de la novela y contar qué es de la relación entre Lecter y Clarice (Berstein, 2015). Después, Fuller rebajó sus expectativas a solo seis temporadas y además se planteó incluir en la serie - y variarlos - hechos de la juventud de Lecter provenientes de Hannibal Rising, hasta que la NBC, que emitía la serie, decidió no renovarla, por lo que concluye de ese modo con los sucesos relatados en El Dragón rojo - aunque también acaban por incluirse en esta temporada los elementos variados sobre la juventud de Lecter, e, igualmente variados, algunos elementos presentes en Hannibal, como la estancia en Florencia de éste- ${ }^{11}$.

A raíz de la serie — curiosamente no tanto de las películas, aunque posiblemente éstas hayan sido más exitosas en términos de público-, han aparecido algunas publicaciones relacionadas con el personaje. Por ejemplo, existe un libro oficial sobre la serie: The Art and Making of Hannibal: The Television Series de Jesse McLeen (Titan Books, 2015); también otras guías no autorizadas, tanto de la propia serie como del personaje ${ }^{12}$. Feeding Hannibal. A Connoisseurs Cookbook, de la estilista y

\footnotetext{
${ }^{9}$ Por cierto que, en su momento, ya la propia novela Red Dragon había sido objeto de un tipo de trasvase medial poco estudiado: ante su éxito, se había resumido y serializado para la revista Crime Digest (Simpson, 2010: 13). Sobre la práctica hipertextual del digest, vid. Genette, 1989: 316-317.

${ }^{10}$ https://en.wikipedia.org/wiki/Hannibal_TV_series).

${ }_{11}$ En la actualidad se especula con que Fuller pueda concluir la saga con una cuarta temporada compuesta de unos pocos capítulos de mayor extensión, a modo de miniserie donde explorar lugares y momentos de El silencio de los corderos (Galluzzo, 2016).

${ }^{12}$ The Hannibal Files: The Unauthorised Guide to the Hannibal Lecter Phenomenon, de Daniel O’Brien (2009); Spoiler Alert: Hannibal Season One: An Unauthorized Critical Guide, de Paul Brian McCoy (2015).
} 
experta en cocina Janice Poon (Titan Books, 2016), un libro con las recetas de cocina que prepara Lecter en la serie. Hannibal Lecter and Philosophy: The Heart of the Matter de Joseph Westfall (Open Court, 1025), en la que aprovecha la figura de Lecter para realizar divulgación filosófica ${ }^{13}$. La marca de juguetes Funko, cuya colección POP! consiste en muñecos caricaturizados de las principales series y franquicias de éxito, tiene figuras de Hannibal y Will Graham con diversos accesorios —caracterizados según la serie- También hay al menos dos páginas de fans destinadas a dar cuenta minuciosa del personaje a través de las novelas, películas y la serie: una, en inglés, y otra, en castellano ${ }^{14}$; en ambas pueden encontrarse sinopsis, referencias cruzadas, citas, alusiones, fotografías, curiosidades, y en general, el tipo de información colaborativa por parte de los fans. Por último, también encontramos en internet una amplia muestra tanto de fandom como de fanart exclusivamente relativo a Hannibal -en el caso del fanart, claramente orientado hacia la serie y los actores que encarnan a los personajes en ésta- ${ }^{15}$.

\section{3. ¿Es Hannibal un relato transmedia? Discusión del problema}

En esta descripción del devenir de Hannibal Lecter como personaje de ficción, hemos podido comprobar que éste existe tanto en novelas como en películas y en una serie para la televisión. ¿Convierte esto al relato de Lecter en un producto transmedia? El estatuto específico de la transmedialidad frente a cualesquiera otros tipos de remediación o circulación de una historia a través de distintos medios es una de las discusiones más características a la hora de abordar las relaciones entre narrativa y medios. Desde las definiciones de transmedialidad más estrictas, que subrayan que se trata de una práctica específica que da lugar a productos concretos, a las más laxas, que incluso parten del principio de que toda narración es, en sí misma, transmedial (Thon, 2016: pos. 261), podemos encontrar todo un abanico de posiciones intermedias. A continuación, vamos a examinar en detalle el recorrido realizado por el mundo de Hannibal para confrontarlo con alguna de estas definiciones y posiconamientos con objeto de intentar responder a la pregunta; de paso, esperamos ofrecer algún sesgo novedoso sobre el proceso de trasvase intermedial.

Los primeros avatares del personaje de Hannibal Lecter pertenencen al dominio de lo puramente textual y narrativo: son prolongaciones que realiza el autor de la novela original, Thomas Harris, en novelas sucesivas: lo que Genette ha denominado «prolongación autógrafa» de un texto (1989: 201) «para conducirlo más allá de lo que inicialmente se consideraba su término» (1989: 253-254) y «cuyo móvil es, en general, el deseo de explotar un primer o un segundo éxito» (1989: 254). Nos encontramos ante la ampliación de una historia a través de un único medio. Eso sí: en cuanto creación y prolongación

\footnotetext{
${ }^{13}$ No es el primer libro que emplea películas y series de éxito para hablar sobre historia de la filosofía. Otros ejemplos, de entre muchos posibles a elegir: The Simpsons and Philosophy, de William Irwin et alii (Open Court, 2001); The Matrix and Philosophy también de Irwin (Open Court, 2004); The Sopranos and Philosopy, de Richard Greene et alii (Open Court, 2004); no cuesta trabajo comprender que se trata de toda una colección de una misma editorial.

${ }^{14}$ Hannibalpedia FANDOM: http://es.hannibal.wikia.com/wiki/Hannibalpedia y Hannibal the Wiki: http://hannibal.wikia. com/wiki/Hannibal_Wiki

${ }^{15}$ Vid. https://fanlore.org/wiki/Hannibal_(TV_series) y Baker-Whitelaw (2013).
} 
de un mundo que se expande poco a poco más allá de una novela — aunque sea en otras- y, en este caso, da un mayor protagonismo y caracteriza más en profundidad a ciertos personajes $-\mathrm{y}$ a Lecter en particular-, esta prolongación textual sienta las bases de su futura recreación, amplificación y enriquecimiento transmedial - como es el caso de otras sagas de novelas que luego han devenido en fenómenos transmedia, como las del mundo de Harry Potter, por ejemplo, que también comenzaron siendo prolongaciones en forma de novela de la novela original (vid. Saldre y Torop, 2012)—. Si, en palabras de Freeman, la narrativa transmedia tiene mucho de construcción de un edificio al que se le van realizando sucesivas ampliaciones (Freeman, 2017: 26), podríamos decir que un relato que se se expande a través de diversas novelas está estableciendo los cimientos de esa futura construcción.

Tony Williams ha percibido en las novelas de Harris, debido a su reflejo violento y feísta de los aspectos más oscuros de la sociedad contemporánea sumado a una popularidad que las convierte en obras de culto, una cualidad de «Pulp fiction» (2008: 183). Dicha cualidad nos permite comprender el potencial transmedia del mundo de Hannibal Lecter ya que, según Scolari, Bertetty y Freeman, una de las características de la pulp fiction es - entre otras-, aquello que Steirer denomina «narrative expansion»: «for readers, the texts [...] grew into stepping stones of entertainment - the narrative fruther expanding across texts» (2014: 9). De este modo,

[...] narrative expansion involves the expansion of a given narrative beyond the confines of a single text, usually in such a way that mahyo of the original characters, settins and histories are peserved and/or futher developed throuhg encounters with new characters, settings and events (Scolari, Bertetty y Freeman, 2014: 9).

Que no es otra cosa sino lo que le ocurre al personaje Lecter, sobre todo a partir de la novela Hannibal. En esta característica percibimos ya, según los atuores, un impulso transmedia, que se percibe sobre todo en obras pulp más características del siglo XX — como, por ejemplo, el Tarzán de Edgard Rice Borroughs - que pueden considerarse antecedentes directos de la narrativa transmedia, si no directamente tales.

De forma simultánea a la expansión del mundo de Hannibal a través de las novelas, aparecen las dos primeras películas, que son adaptaciones cinematográficas de las novelas: Manhunter de Red Dragón, y The Silence of the Lambs del libro homónimo. En relación a si pueden considerarse las adaptaciones cinematográficas o no como prácticas transmediales, Scolari (2012: 54-56) ha resumido el núcleo en que puede enmarcarse el debate ${ }^{16}$ : para algunos autores, si la narrativa no se expande, esto es, no se amplía, no se produce transmedialidad. En las adaptaciones, en cuanto práctica de traducción intermedial o intersemiótica según la entiende Jakobson como «interpretation of verbal signs by means of signs of nonverbal sign systems» (Saldre y Torop, 2012: 32) no se produce esta expansión. Scolari resume esta postura con una cita de Long: «Retelling in a different media type is adaptation while using multiple media types to craft a single story is transmediation» (Scolari, 2012: 54; vid. Freeman, 2017: 21). No obstante, esto puede ponerse en cuestión en la medida en que una adaptación no supone

\footnotetext{
${ }^{16}$ Para una reflexión sobre la delimitación terminológica de carácter más epistemológico, puede consultarse Sánchez-Mesa y Baetens, 2017, 7-11.
} 
una translación completamente redundante de una misma historia, sino que incorpora aspectos o puntos de vista que, por lo general, la matizan y enriquecen, y por tanto, de algún modo sí contribuyen a expandir la historia (Scolari, 2012: 54-55). En este sentido, lo mejor, según indica Jenkins, es percibir «adaptation and extension as part of a continuum in which both poles are only theoretical possibilities and most of the action takes place somewhere in the middle» (Scolari, 2012: 55). Por su parte, Saldre y Torop (2012: 32-33) consideran, en un sentido análogo al que nosotros hemos indicado al hablar de las propias expansiones (intra)textuales de una misma historia — por ejemplo en forma de novelas sucesivas - , que las adaptaciones contribuyen a cimentar la historia transmedia — presente o futuraen su conjunto, en la medida en que sirven, a través del cotejo de sus distintas variaciones -inherentes, añadimos nosotros, a la especificidad del medio-, para percibir las invariantes del mundo que se está construyendo y que constituyen su esencia.

Por lo que respecta al caso que nos ocupa, en nuestra opinión, en este estadio no puede hablarse todavía de transmedialidad en el mundo de Hannibal, sino tan solo de intermedialidad porque, en efecto, no parece haber una voluntad de expandir la narración en ningún sentido, sino tan solo remediarla con objeto de llegar a otras audiencias, en un proceso habitual de adaptación cinematográfica $^{17}$. No obstante, también creemos que, en el caso de la película El silencio de los corderos, aunque no se produzca en ella una expansión de la historia, ésta sí desempeña un papel fundamental en la constitución de Hannibal como un personaje transmedial: la caracterización de Anthony Hopkins y el éxito de la película son determinantes para percibir el potencial expansivo del personaje. Nunca sabremos hasta qué punto este éxito animó a Harris a escribir una continuación donde Lecter tuviera tanto protagonismo; lo que sí sabemos es que ya el guion de la tercera adaptación, la de dicha continuación, Hannibal, comenzó a hacerse sobre el manuscrito inédito de la novela, según hemos dicho, con lo que empieza a intuirse un principio de coordinación entre los medios - la novela casi parece escribirse para ser llevada al cine, o al menos el autor tiene plena conciencia de que va a suceder-. En la película, además, ya se busca una coherencia con la anterior en la elección de los protagonistas - fallida en el caso del personaje de Clarice Starling-. Por su parte, en esta novela aparecen referencias a la infancia de Lecter y al trauma que marcará su vida en forma de recuerdo del propio Lecter narrado en cursiva, que es casi un anticipo o fragmento textual de Hannibal Rising (Harris, 1999: 278-279) ${ }^{18}$. Harris está hablando de elementos de la historia que, aunque aún no existen de forma textual -o material - ya pueblan su mundo: constituyen el pasado efectivo del personaje. A estas alturas, Thomas Harris ha creado una serie de novelas que se pueden leer de forma independiente pero que incluyen referencias unas a otras y que cobran su pleno sentido si se conocen; no ha habido salto de medio, pero el potencial transmedia está ahí.

\footnotetext{
${ }^{17}$ En este sentido, Scolari señala cómo desde el punto de vista del productor «the distances between narrative expansion and adaptation become shorter. For Hollywood studios transmedia storytelling and adaptation are nothing more than two different textual strategies that share teh same goal: to increase the commerical exploitation of a charcaher or a fictional universe» (Scolari, 2012: 56).

${ }^{18}$ Antes, en la misma novela, Harris sugiere una suerte de spoiler avant la lettre de la novela aún no escrita cuando alude a que Lecter sueña con su hermana Mischa, «muerta y digerida hace mucho tiempo» (1999: 202).
} 
No obstante, la nueva adaptación o remake de Red Dragon sí que consideramos que marca un punto de inflexión en un proceso que, a nuestro juicio, va del mero trasvase intermedial a un principio de transmedialidad y, en el continuum mencionado por Jenkins, comienza a aproximarse a ésta. Aunque Red Dragon se trate de nuevo de una adaptación, en ella se producen algunos cambios significativos en relación a la novela que apuntan hacia la construcción de un mundo coherente y la posibilidad de su ampliación; la consolidación de una coherencia en la historia que ya se apuntaba en Hannibal. En este momento surge el concepto que marca el punto de inflexión en nuestra indagación de Hannibal como fenómeno transmedia: franquicia. Hannibal Lecter se ha convertido en una franquicia, y así lo entiende Dino de Laurentiis quien, exasperado por la lentitud con la que Harris ultima sus novelas y pensando ya en una nueva entrega ${ }^{19}$, dirá, en un tono muy poco diplomático, algo muy significativo para el problema que nos ocupa: «I own [the movie rights to the] character Hannibal Lecter... I say to Thomas, “If you don't do [the prequel], I will do it with someone else... I don't want to lose this franchise [el énfasis es nuestro]. And the audience wants it"» (Simpson, 2010: 28). Una franquicia es una de las maneras principales de extender productos y servicios en una cultura orientada hacia el consumidor (Freeman, 2017: 113). De acuerdo con Pratten, se trata de un proyecto transmedia característico: «It's a series of single-platform deliverables - a book, a movie, a game. In many ways, the platforms are independent except that they otften cover different narrative spaces: prequel, sequel, flashback which may dictate a release order or schedule» (2011: 13). En general, todas las plataformas —novela, película... - tienen la misma importancia y la recepción por parte del espectador suele ser de adición (Pattern, 2011: 18).

Desde este punto de vista, en Red Dragon hay un esfuerzo por acrecentar el protagonismo de Lecter frente a su carácter secundario en la novela - y en la primera adaptación-: por ejemplo, lo que en la novela es una carta que Lecter le remite a Graham, según vimos, en la película se convierte en una segunda entrevista que incrementa la presencia personaje. Con todo, lo más significativo es el comienzo de la película, con un prólogo a modo de flashback donde el personaje de Lecter es el absoluto protagonista y donde se hace hincapié en sus cualidades más reconocibles: cultura, refinamiento y canibalismo ${ }^{20}$. Pero, sobre todo, se nos muestra el momento de su captura por parte de Graham - tanto en la novela como en Manhunter este se relata por el propio Will en forma de diálogo-.

En la historia, tal y como está planteada por Harris en El dragón rojo, Will Graham atrapa de forma fortuita a Lecter cuando va a interrogarlo porque una de las víctimas ha sido paciente suyo. Ve unos libros antiguos de medicina que tiene Lecter y los asocia con un grabado que recuerda haber visto en libros semejantes, El hombre herido, que Lecter había recreado en sus crímenes (Harris, 1992: 75 76). Lecter comprende que Graham lo ha sabido e intenta matarlo para protegerse. No obstante, en la adaptación cinematográfica, la situación se presenta de forma que Graham y Lecter han estado

\footnotetext{
${ }^{19}$ Y ello a pesar de que El dragón rojo, sin ser un fracaso, tampoco fue un blockbuster, aun cuando Harris había dicho antes de su estreno que era la mejor adaptación de una de sus obras (Simpson, 2010: 27).

${ }^{20}$ En este prólogo, ha matado a un flautista de la sinfónica de Baltimore porque desafina y luego ofrece un suntuoso banquete a la directiva de la orquesta con las mollejas del músico.
} 
trabajando juntos en la resolución del caso; como si Lecter estuviera prestando asesoría psicológica a Graham - lo que no se dice pero se deduce de la conversación que mantienen-. Entonces Graham va a visitar a Lecter para contarle que de pronto ha comprendido que el asesino se come las visceras de sus víctimas, y el libro que ve al ausentarse Lecter un momento es el Larousse de cocina con una entrada sobre mollejas subrayada. Más allá del trueque de libros, lo verdaderamente importante del cambio es que establece una relación de colaboración y amistad entre Lecter y Graham que subraya la dualidad entre ambos — que son las dos caras de una misma moneda; que comparten cualidades y pulsiones pero que uno las ha sublimado hacia el bien con un gran coste personal de angustia y culpaY este mismo punto de partida es el que toma la serie desde su mismo comienzo, que nos cuenta el origen de esa relación en el hecho de que Jack Crawford encarga a Lecter -eminente psiquiatra de la comunidad - la evaluación psicológica de Graham porque lo necesita en el cuerpo pero teme su inestabilidad emocional y, a partir de ahí, estos se hacen amigos y comienzan a trabajar juntos. En la serie, Will no atrapará a Lecter hasta la tercera temporada y, mientras, asistimos a la evolución de esta amistad en la que además Lecter está colaborando activamente con el FBI en la resolución de casos, lo que le permite distorsionar y desestabilizar las investigaciones como el zorro que guarda un gallinero. Es decir, se ha producido una variante, pero también una ampliación verosímil a partir de la historia original en la serie, cuyo origen está, no obstante, en una de las adaptaciones cinematográficas, que ya ha comenzado el proceso de enriquecimiento del relato. Aquí, el productor ha desplazado al autor en la fijación y ampliación de los hechos sucedidos en la trayectoria del personaje de forma coherente con el progresivo establecimiento, ya mencionado, del personaje como franquicia.

Otra búsqueda de cohesión importante que se produce en el remake es utilización de la celda de Lecter y del propio psiquiátrico de Baltimore donde está confinado que aparecían en la película $E l$ silencio de los corderos como una marca deliberada de continuidad con ésta. De hecho, MGM no quería ceder a De Laurentiis los derechos de imagen del hospital y, a cambio de su asentimiento final, entró en la producción como distribuidora internacional de la película (Simpson, 2010: 25). Pero el ejemplo más claro de búsqueda de encaje se produce en el epílogo añadido en la película, que tampoco se encuentra en la novela: volvemos a la celda de Lecter — nueva presencia del personaje-, y el director del hospital le avisa de que tiene una visita: una joven del FBI que viene a hacerle unas preguntas en relación a un caso. Evidentemente, es un guiño al espectador que sabe que se trata de Clarice Starling: una referencia a una película anterior pero que, cronológicamente, cuenta una historia posterior a la que estamos viendo. En la novela este guiño es imposible porque la continuación aún no exisitía y es posible que Harris ni siquiera hubiera pensado en ella: el mundo de Lecter va tomando forma. La impresión de relato coherente y ampliado en el contexto de una franquicia se acrecienta con la aparición de Hannibal Rising, que viene a contarnos la infacia y juventud de Lecter y el origen traumático de su psicopatía. Seguimos dentro de un ciclo novela-adaptación cinematográfica pero, como vimos, ya hay una simultaneidad entre el guion y la película, así como estrategias multimedia coordinadas de lanzamiento del producto - aparte de ampliar, en cuanto relato, algún esbozo que se apuntaba en la novela anterior, Hannibal, como ya se ha mencionado-. 
En definitiva, a partir del remake de Red Dragon comienza a nuestro juicio, un proceso paulatino y a posteriori de consideración de los distintos textos en que aparece Hannibal como un único mundo que se va cohesionando bajo la estructura de una franquicia. Es decir: se va produciendo poco a poco un proceso -insistimos que a posteriori- de convergencia entre las partes y los medios cuya relación, hasta ese momento, había resultado más azarosa. A partir de Jenkins (2008), la idea de convergencia entre distintos medios por los que el contenido fluye es fundamental para comprender la noción de transmedialidad. Según Freeman, «convergence itself seems to denote multiple pre-existing elements of media content all coming together» (2017: 22). Y para esta labor de encaje y limado de piezas que en algunos casos no estaban preparadas del todo para encajar, desempeñan una labor fundamental los paratextos, que ejercen de argamasa desde fuera del texto. Según Genette, los paratextos, esto es, los textos que rodean al texto principal y sirven para presentarlo constituyen «a privileged place of a pragmantics and a strategy, of an influence on the public, an influence that [...] is at the service of a better reception ofr the text and a more pertinent reading of it (more pertinent, of course, in the eyes of the author and his allies)» (1997: 2). En realidad, van más allá: dan forma y construyen la propia narrativa al orientar su interpretación (Evans, 2011: 19). En el caso que nos ocupa, si, por ejemplo, la cubierta de la primera edición de Red Dragon estaba diseñada a partir del grabado The Red Dragon and the Woman Clothed in Sun de William Blake - referencia fundamental en la novela, como puede deducirse de su título-, a partir del remake la cubierta pasa a tener el cartel de la película. Esta práctica es habitual en las relaciones intermediales de adaptación pero, en este caso, el cartel de la película es el rostro de Hopkins caracterizado como Lecter; a partir de aquí, esto va a ser una constante en las reediciones de las novelas —incluida The Silence of the Lambs, donde se sustituye la polilla, tan icónica, sobre la boca de Clarice-: el rostro de Lecter se convertirá en la imagen de la franquicia - luego sucederá también en la serie, ahora con el rostro de Mikkelsen-. Además, en las reediciones de Red Dragon se incluirá la frase promocional: «The Terrifying classic that introduced Hanibal Lecter» con el nombre de Lecter en un cuerpo de texto mayor. Incluso en el caso del texto menos convergente, Manhunter — que, no olvidemos, pertenece a la misma productora-, a partir de ese momento, en las reediciones del DVD, incluye en su carátula un paratexto que dice «Hannibal Lecter's legacy of evil begins here» ${ }^{21}$.

Tras Hannibal Rising, novela-película surgidas simultáneamente, el productor De Laurentiis, consciente de la lentitud creativa de Harris, vuelve a manifestar su impaciencia por disponer de nuevo material para la franquicia. Sus declaraciones nos proporcionan una nueva pista sobre cómo Hannibal Lecter ha pasado de ser el personaje de una novela a un personaje de una franquicia transmedia: «Another book may take another ten years. But we can ask Thomas Harris if he'd give some

\footnotetext{
${ }^{21}$ En español apareció como: «la primera película de la saga de Hannibal Lecter». En la primera edición de bolsillo de $E l$ dragón rojo —citada en la bibliografía —, aun antes del remake — pero después de El silencio de los corderos-, ya aparece en cubierta la imagen de un personaje cubierto con una máscara protectora —en una alusión clara- y el subtítulo: «el primer caso de Hannibal Lecter». Por cierto, que el título The Silence of the Lambs se tradujo en español en las primeras ediciones de la novela un tanto incomprensiblemente como El silencio de los inocentes. Después del éxito de la película, obviamente se cambió. Nótese que estos cambios no están necesariamente coordinados, sino que son procesos espontáneos de convergencia por motivos comerciales.
} 
cooperation for the storyline, because if you have the right storyline and then there's a script, then you can have Hannibal Lecter» (Simpson, 2010: 27); es decir: lo importante es la historia, sus líneas maestras; si se tiene, se tiene lo que se busca, que es el personaje, Hannibal Lecter. De Laurentiis, en efecto, no está dispuesto a esperar, y acaba confiando a otra persona la ampliación de la historia de Lecter a partir de Red Dragon, cuyos derechos posee, y Hannibal, la serie, ve la luz de la mano de Bryan Fuller en lo que Genette define como «prolongación alógrafa» — por contraposición a la autógrafa-, cuyo objetivo es no matar la gallina de los huevos de oro cuando el autor ha decidido hacerlo (Genette, 1989: 255); solo que Genette no contemplaba la posibilidad de esta prolongación $a$ través de otro medio. Es aquí a nuestro juicio, donde el personaje de Hannibal adquiere una cualidad plenamente transmedia. No solo porque haya dado el salto a un tercer medio, sino porque, por primera vez, no se trata de una adaptación, sino que se están explorando partes desconocidas de la historia y del personaje, en ese nuevo medio, lo que es característico del transmedia: «not as versions of the same story but rather as extensions of the same story» (Freeman, 2017: 33). Esto es, de hecho, lo que movió a Bryan Fuller a crear la serie. Busca contar, según sus propias palabras, «the missing chapter» (Goldman 2013), cuando, pasada su juventud, Hannibal es tanto un psiquiatra como un caníbal en ejercicio:

We had seen the prequel Hannibal Rising, when he was a young man around World War II, and certainly post-incarceration. But the unexplored chapter of the Hannibal Lecter story that doesn't exist in literature or film or on television [el énfasis es nuestro] is when he was a practicing psychiatrist and cannibal (Goldman, 2013).

En esta prolongación de la historia, asismismo, el papel de Harris se ha trasvasado a Fuller, en la medida en que él ya no crea estos nuevos episodios de la vida de Lecter que vienen a colmar los huecos de su historia. No obstante, en los títulos de crédito aparece que la serie está basada en los personajes de El dragón rojo de Thomas Harris. Otra de las características de las narraciones transmedia es la tensión entre un autor centrífugo que garantiza la coherencia — convergencia- del relato y la participación de otros agentes —incluidos los propios espectadores - que coparticipan en el proceso de ampliación — transmedial— del relato (vid. Freeman, 2017: 32-33). En este sentido, el nombre de un autor, como ha señalado Freeman, puede implicar, a través de paratextos como los señalados - la mención del autor en los títulos de crédito- una market-author function donde «the mere presence of an author's name on a media text (i. e. on the cover of a book, on the poster for a film etc.) can point audiencies across multiple media to other media texts that constitute a story world» (Freeman, 2017: 37) por un efecto de asociación ${ }^{22}$. También existirán tensiones con los derechos de autor: Fuller no puede utilizar a Clarice Starling y otros personajes de El silencio de los corderos - problemas de una transmedialidad sobrevenida y no diseñada desde el comienzo- (Goldman,

\footnotetext{
${ }^{22}$ En este sentido, resulta muy significativo lo que dice Harris en el prólogo, ya mencionado a la reedición de Red Dragon de 2000 (pos. 156): «By the time I undertook to record the events in Hannibal, the doctor, to my surprise, had taken on a life of his own. [...] In the end [...] you must let characters go».
} 
2013) ${ }^{23}$. Además, es el momento en el que comienza a haber merchandising y a desarrollarse el fan art, fan fiction, foros, etc. característico de la cultura participativa del transmedia ${ }^{24}$.

Fuller, además, no solo amplía la historia, contándonos nuevos sucesos, sino que amplía además el campo de interpretación y posibilidades: ya hemos hablado más arriba de cómo aquí Will Graham y Lecter en realidad son colaboradores —el héroe y su némesis juntos-; además, al pensar en desarrollar la parte correspondiente a El silencio de los corderos, donde ya no aparece Graham, Fuller pensaba incluirlo como personaje y contar cómo recupera tanto su salud como la relación con su mujer (Goldman, 2013). Además, aparte del hecho evidente de que los personajes principales estén interpretados por otros actores, introduce cambios de sexo en algunos personajes ${ }^{25}$. La acción sucede en la actualidad: Lounds no es reportera, sino que escribe en un blog sensacionalista; Lecter utiliza un iPad...

No obstante todas estas incoherencias e inestabilidades - que curiosamente alteran la época en que suceden los hechos, pero no así la cronología ficticia-, seguimos pensando que el relato de Hannibal Lecter construido de forma transmedial se mantiene razonablemente estable gracias al hecho clave de que no está sustentado tanto en un mundo ficcional - aunque también- cuanto en el propio personaje: se trata de un caso evidente de character-building transmedia (vid. Rosendo, 2016; Freeman, 2017). Según la definición de Bertetti, esto se produce cuando «a transmedia character is a fictional hero whose adventures are told in different media platforms, each one giving more details on the life of that character» (Rosendo, 2016: 23). Como señala Nieves Rosendo, se trata de un concepto «that goes beyond the idea of character belonging to a transmedia narrative» (2016: 25): en realidad, más que pertenecer — que pertenece-, la articula. En este caso, «it was character, then, that worked to build narrative references between the films and the books, connecting both media texts as components of a larger storyworld» (Freeman, 2017: 24). Al igual que en el de otros personajes de ficción característicos como Sherlock Holmes —con cuya iconicidad, según vimos, comparaba Lanchenster la de Lecter-, y a diferencia de narraciones claramente construidas en torno a un mundo - como, paradigmáticamente, Star Wars - es el carisma y la fascinanción por personaje los que sostienen el relato transmedia y sobre el que el espectador busca saber — de su pasado, etcétera- a través de los distintos medios. ¿Y cómo supera el personaje ese trasvase a distintos medios y, dentro de estos, incluso a distintas épocas y a ser encarnado por actores diferentes? Doležel nos da una pista: «el hilo que mantiene unidas todas las encarnaciones de un individuo en todos los mundos posibles [aquí cabría decir medios] es delgado y teóricamente controvertido: el nombre propio como designación rígida» (1999: 38). El personaje, además, tiene unas características, físicas, morales, etcétera, reconocibles invariantes, imprescindibles para el reconocimiento; no obstante, además, cada

\footnotetext{
${ }^{23}$ Para las relaciones problemáticas entre transmedia y copyright, puede consultarse, por ejemplo, Freeman (2017: 117$118,135-136)$.

${ }^{24}$ Acerca de las relaciones entre merchandising y transmedia, pueden consultarse Freeman (2017: 128-131) y Scolari (2012: 261-262). Para la cuestión de los contenidos generados por los usuarios, Scolari (2012a: 243-250).

${ }^{25}$ Por ejemplo, Alan Bloom, agente amigo de Graham, es ahora Alana Bloom, y otro tanto sucede con Freddy Lounds, el periodista sensacionalista del Tattle, que se convierte Frederica, Freddie, Lounds, una mujer - aunque, curiosamente, también pelirroja, como el periodista en la novela y la adaptación originales-.
} 
sucesiva encarnación va añadiendo características nuevas que se amalgaman con las precedentes (Rosendo 2016: 23). Poco a poco, el personaje se va encarnando así en la cultura popular y volviéndose un mito: en el caso de Hannibal Lecter, el refinamiento, la mirada inquisitiva, las cualidades perceptivas cuasi sobrenaturales, etcétera. En las narrativas transmedia fundadas en el personaje ocurre no solo que el personaje resulta reconocible en sus distintas variantes mediáticas, sino que incluso es reconocido, aun superficialmente — como sucede con Don Quijote o Drácula—, por quienes no están familiarizados con ninguna.

\section{Conclusión}

El relato de Hannibal, aunque es evidente que no surgió como una realidad transmedia, se ha ido configurando como tal a partir del éxito que fue adquiriendo su personaje protagonista conforme se adueñaba de las distintas narraciones. Esta transición culmina cuando la saga de Hannibal se convierte en una franquicia por el impulso de Dino de Laurentiis, dueño de los derechos sobre Lecter, aunque se realice en buena medida con la colaboración del autor del personaje, Thomas Harris. «Hannibal» puede considerarse a novel-based franchise (Thon, 2016: pos. 238) que ha pasado por todos los estadios crossmedia característicos: «narrative works [which] are part of an intramedial series, an intermedial adaptation process, and/or a transmedial enternatinment franchise» (Thon, 2016: pos. 320). Si bien no alcanza los niveles de sofisticación de otras narraciones transmedia en lo que respecta a diversidad de medios, convergencia entre estos y participación del público - aunque, como hemos visto, sí existe un nutrido muestra de fan art y fan fiction que desarrolla narraciones al margen de los textos canónicos-, creemos que se trata de un ejemplo significativo de cómo una obra narrativa propia del siglo XX se va adaptando a los medios y modos de contar del XXI: una narración transmedia que va tomando conciencia de sí misma y donde podemos percibir la importancia de los paratextos —cubiertas de libros, fajas, carátulas de películas, eslóganes- para la construcción de una convergencia $a$ posteriori de elementos; por seguir con la metáfora de Freeman de la narrativa transmedia como la construcción de un edificio, en lugar de tratarse de uno diseñado desde el principio, ha habido que ir ajustando sus diversas habitaciones, y aquí los paratextos son los que, al menos de fachada para fuera, han limado las asperezas y unificado el enlucido. Como indica precisamente Freeman citando a Fast y Örnebring, es necesario también entender el transmedia storytelling «in terms of the "accured characteristics that are more ad hoc/contingent than planned"» (Freeman 2017: 22); y añade: «rather than limiting conceptions of transmedia storytelling to "planned, strategic aspect of creation", it is equally as important to "emphasise the many disjunctions and contradictions that almost inevitably follow when extending transmedia worlds across/between media"» (2017: 22). No obstante, más allá de los paratextos, la fuerza cohesiva del relato emana de la fascinación que ejerce la figura mítica de Hannibal Lecter, con lo que nos encontramos con una narración transmedia sustentada en la fuerza de un personaje, lo que vincula además de forma coherente a esta franquicia con algunas narraciones pulp del pasado - algunas erigidas en franquicia también - cuya coherencia a través de espacios y tiempos 
irregulares se mantenía igualmente por el poder de seducción que un ente de ficción conseguía suscitar entre sus seguidores.

Hannibal Lecter, para mitigar su encierro y su soledad, ha construido un portentoso palacio de la memoria donde almacena todos sus recuerdos. Se nos explica en Hannibal:

El palacio de la memoria era un sistema mnemotécnico bien conocido por los sabios del mundo antiguo [...]. Como los eruditos que lo precedieron, el doctor Lecter almacena un asombroso cúmulo de datos asociados a objetos de estas mil estancias; pero, a diferencia de los antiguos, su palacio cumple una segunda función: a temporadas, le sirve de residencia (Harris, 1999: 274).

En el comienzo de Hannibal. El origen del mal (2007: 7), se abunda en la descripción:

La puerta del palacio de la memoria del doctor Hannibal Lecter se encuentra entre las sombras que pueblan su mente, y su picaporte solo puede encontrarse a tientas. Este curioso pórtico conduce a espacios vastos y bien iluminados de principios del Barroco, a pasillos y cámaras que rivalizan en número con las del museo Topkapi.

Las vitrinas lo ocupan todo, espaciosas y bien iluminadas, guardan bajo llave recuerdos que evocan otros recuerdos en progresión geométrica.

Después se nos advierte de que, en esta novela, se va a acceder a la parte más inquietante e ignota del palacio: el sótano, donde se albergan los recuerdos de su infancia: «Busquemos juntos el picaporte aquí, en la calurosa oscuridad de su mente. Una vez lo encontremos [...], sin mirar ni a derecha ni a izquierda, dirijámonos a la Sala del Principio, donde las vitrinas contienen, en su mayoría, piezas fragmentarias» (Harris, 2007: 8).

Quizá la narración transmedial de la historia de Hannibal Lecter no sea tanto «like extensions added on to a house that never stopped being built», según la definición que hace Freeman del transmedia storytelling (2017: 111), sino un remedo del complejo y barroco palacio de la memoria que él mismo ha ido construyendo y que, en cuanto auxiliar de la memoria, es engañoso como ella. Eso explicaría que, conforme va expandiéndose, surja cierta confusión en la apariencia -incluso en el sexo- de algunas de las figuras que lo pueblan; que algunos elementos sean extrañamente recurrentes o que se contradigan. No solo la memoria es engañosa: Lecter, además, es un embustero que manipula fechas y declaraciones «para confundir a las autoridades y a sus biógrafos» (Harris, 2007: 8). Saldre y Torop (2012) han definido como una de las características de la transmedialidad su cualidad espacial y su vinculación con la memoria: «Meanwhile, in the memory of the reader who is familiar with the novels, the movies [...] and other subtexts, it is impossible to distinguish which aspects of the mental whole originate in which particular subtext» (2012: 31). Quizá no solo el mundo de Hannibal Lecter, sino en general los mundos transmedia sean como un palacio de la memoria donde «el espectador se abisma en el espectáculo; se pierde para siempre en lsa tramoyas infitias; lee durante un tiempo ya inmedible, los hilos de los tapices de los telones; pregunta a las carátulas: cada gesto, suscita una emoción...» (Rodríguez de la Flor, 1996: 16). 
Tropelías. Revista de Teoría de la Literatura y Literatura Comparada, 28 (2017)

José Manuel Ruiz Martínez

\section{Referencias bibliográficas}

BAKER-Whitelaw, G. (2013): «A delicious guide to "Hannibal” fandom on Tumblr», The Daily Dot, en https://www.dailydot.com/parsec/fandom/guide-hannibal-tv-fandom-tumblr-fannibals (última consulta, 15-7-2017).

BersteIn, A. (2013): «Exclusive Interview: Hannibal News on Season 1, Season 2 and beyond from showrunner Bryan Fuller», Assigment X, en https://www.assignmentx.com/2013/exclusiveinterview-hannibal-news-on-season-1-season-2-and-beyond-from-showrunner-bryan-fuller (última consulta, 15-7-2017).

Evans, E. (2011): Transmedia Television. Audiences, New Media and Daily Life. New York, Routledge.

Freeman, M. (2017): Historizing Transmedia Storytelling. Early Twentieth-Century Transmedia Story Worlds. New York, Routledge.

Galluzzo, R. (2016): «Shock Waves, Episode 31: For The Love Of Horror, With Bryan Fuller!», Blumhouse, en http://www.blumhouse.com/2016/12/19/shock-waves-episode-31-for-the-loveof-horror-with-bryan-fuller (última consulta, 15-7-2017).

Genette, G. (1989): Palimpsestos. La literatura en segundo grado. Trad. Celia Fernández Prieto. Madrid, Taurus.

- (1997): Paratexts. Thresolds of Interpretation. Trad. Jane E. Lewin. Cambridge, Cambridge University Press.

GoldMAN, E. (2013): «Hannibal: How Bryan Fuller approached the iconic character», IGN-US., en http://www.ign.com/articles/2013/04/04/hannibal-how-bryan-fuller-approached-the-iconic-cha

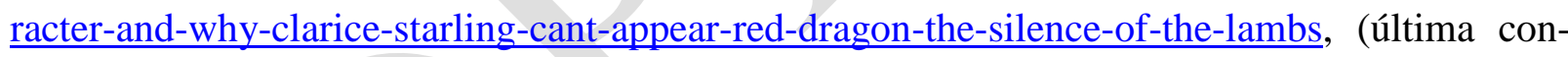
sulta, 15-7-2017).

HARris, T. (1991): El silencio de los inocentes. Trad. Montserrat Conill. Barcelona, Círculo de lectores.

_ (1992): El dragón rojo. Trad. Elisa López Bullrich. Barcelona, Ediciones B.

— (1999): Hannibal. Trad. José Antonio Soriano Marco. Barcelona, Random House Mondadori.

- (2000): Red Dragon. New York, Berkley Books.

- (2007): Hannibal. El origen del mal. Trad. Verónica Canales Molina. Barcelona, Plaza \& Janés.

JENKIns, H. (2008): Convergence Culture: La cultura de la convergencia en los medios de comunicación. Trad. Pablo Hermida Lazcano. Barcelona, Paidós.

PRATTEN, R. (2011): Getting Started with Transmedia Storytelling, en http://www.tstoryteller.com/ getting-started-in-transmedia-storytelling (última consulta, 15-7-2017).

RODRÍGUEZ DE LA FLOR, F. (1996): Teatro y memoria. Siete ensayos sobre mnemotécnica española de los siglos XVII y XVIII. Salamanca, Junta de Castilla y León.

Rosendo, N. (2016): «Character-centered transmedia narratives. Sherlock Holmes in the 21st Century», Artnodes, 18, pp. 20-27. 
SAldre, M. - Torot, P. (2012): «Transmedia Space», en I. Ibrus y C. A. Scolari, eds., Crossmedia Innovations. Texts, Markets, Institutions. Berna, Peter Lang, pp. 25-44.

SÁNCHEZ-MESA, D. - BAETENS, J. (2017): «La literatura en expansión. Intermedialidad y transmedialidad en el cruce entre la literatura comparada, los estudios culturales y los new media studies», Tropelías. Revista de Teoría de la Literatura y Literatura Comparada, 27, pp. 6-27; en https://papiro.unizar.es/ojs/index.php/tropelias/article/view/1536 (última consulta, 15-7-2017).

SCOLARI, C. A. (2012): «The Triplets and the incredible shrinking narrative: Playing in the borderland between transmedia storytelling and adaptation», en I. IBRUS y C. A. SCOLARI,, eds., Crossmedia Innovations. Texts, Markets, Institutions. Berna, Peter Lang, pp. 45-60. (2012a): Narrativas transmedia. Cuando todos los medios cuentan. Barcelona, Deusto.

Scolari, C. A. - Bertetti, P. - Freeman, M. (2014): Transmedia Archaeology. Storytelling in the Borderlines of Science Fiction, Comic, and Pulp Magazines. New York, Palgrave MacMillan.

SiMPSON, P. L. (2010): Making Murder: The Fiction of Thomas Harris. Santa Barbara, ABC-CLIO.

Thon, J.-N. (2016): Transmedial Narratology and Contemporary Media Culture. Lincoln, University of Nebraska Press.

Williams, T. (2008): «From Red Dragon to Manhunter», en B. SzUmskyj, ed., Dissecting Hannibal Lecter. Essays on the novels of Thomas Harris. Jefferson, McFarland \& Co, pp. 102-117. 